В.И. ШАЛАК

\title{
Синтаксическая интерпретация категорических атрибутивных высказываний
}

\author{
Шалак Владимир Иванович \\ Сектор логики, Институт философии РАН. \\ Россия, 119991, Москва, ул. Волхонка, 14, строение 5. \\ e-mail: shalack@gmail.com \\ В статье предложен новый вид моделей для категорических атрибутивных выска- \\ зываний. Эти модели могут быть названы синтаксическими, поскольку субъекту и \\ предикату высказываний сопоставляются не экстенсионалы, а синтаксические опре- \\ деления, посредством которых эти термины вводятся в употребление. Построенные \\ модели свободны от онтологических допущений. Связь между субъектом и предика- \\ том интерпретируется не в терминах теоретико-множественных отношений между \\ экстенсионалами, а в терминах логических отношений между дефиниенсами опре- \\ делений. Доказаны теоремы непротиворечивости и полноты фундаментальной сил- \\ логистики относительно синтаксических моделей.
}

Ключевые слова: атрибутивное высказывание, силлогистика, определение, понятие, аналитичность, модель

События рубежа XIX-XX вв., связанные с проблемами оснований математики, изменили основной тренд развития логики. ФрегеРасселовский подход начал свою победную поступь. Вопросы, дискутируемые ранее в рамках традиционной логики, отошли на дальний план, хотя далеко не на все из них были получены приемлемые ответы. Интерпретация атрибутивных высказываний в терминах одноместного исчисления предикатов и его моделей была признана удовлетворительной и в определенном смысле «закрывала» традиционную логику как самостоятельную область исследований. Вопреки этому логики-философы продолжают обращаться к проблематике традиционной логики. Как ни странно, но даже в такой исследованной вдоль и поперек области еще можно обнаружить много интересного.

Обратимся к моделям простых позитивных категорических атрибутивных высказываний и напомним их стандартную интерпретацию. 


\section{1 Язык}

ОПРЕДЕЛЕниЕ 1. Исходные символы языка

1. $S, P, M, Q, R, N, \ldots$ - символы общих имен;

2. $a, e, i, o-$ силлогистические константы.

ОПРЕДЕЛЕНИЕ 2. Формулы

1. Если $S$ и $P$ - символы общих имен, то $S a P, S e P, S i P, S o P$ - формулы.

2. Ничто другое формулой не является.

\section{2 Экстенсиональные модели}

ОПРЕДЕЛЕНИЕ 3. Экстенсиональной моделъю для простых категорических атрибутивных высказываний будем называть пару $\mathbf{M}=\langle D, \varphi\rangle$, где

1. $D$ - непустое множество индивидов;

2. $\varphi$ - функция, определенная на множестве символов общих имен и сопоставляющая каждому из них некоторое подмножество $\varphi(P) \subseteq D$.

В фундаментальной силлогистике (ФС) истинность атрибутивных высказываний в экстенсиональной модели $\mathbf{M}$ определяется следующим образом:

E.1 $\mathbf{M}=S a P \Longleftrightarrow \varphi(S) \subseteq \varphi(P)$

E.2 $\mathbf{M}=S e P \Longleftrightarrow \varphi(S) \cap \varphi(P)=\emptyset$

E.3 $\mathbf{M}=S i P \Longleftrightarrow \varphi(S) \cap \varphi(P) \neq \emptyset$

E.4 $\mathbf{M}=S o P \Longleftrightarrow \varphi(S) \backslash \varphi(P) \neq \emptyset$

Формула $A$ общезначима, если и только если она истинна в каждой модели $\mathbf{M}$.

Именно такого понимания категорических атрибутивных высказываний придерживается Д. Гильберт в работе [2, с. 73-80]. При этом 
интересно сделанное им замечание «Наше отклонение от Apистотеля... оправдывается потребностями математических применений логики, где класть в основу аристотелево понимание было бы нецелесообразно» [2, с. 79].

Очевидно, что при такой интерпретации атрибутивных высказываний формулы вида $S a S$ будут общезначимыми и аналитически истинными. Высказывания других видов могут быть истинными лишь в конкретных моделях, что соответствует их эмпиричности. Для установления значения таких высказываний в конкретных моделях необходимо знать объемы общих имен субъекта и предиката и иметь возможность установить характер отношения между ними. Теоретически это возможно, но как на практике применить эту теорию к высказыванию «Все люди животные» или «Все кентавры имеют человеческую голову»?

Кроме самих высказываний нас интересует и отношение следования между ними. Аксиоматизировать его можно разными способами. В настоящее время наиболее популярен подход с использованием логики высказываний. Для этого к исходным символам языка добавляют связки логики высказываний и расширяют определение понятия формулы. Затем принимают все аксиомы и правила вывода логики высказываний и добавляют к ним ряд специальных аксиом, относящихся к атрибутивным высказываниям. Понятие вывода соответствует понятию вывода в логике высказываний. Все это достаточно хорошо освещено в литературе [1] и потому не требует подробного изложения.

При таком подходе класс общезначимых формул ФС может быть аксиоматизирован следующим образом:

A.0 Схемы аксиом классического исчисления высказываний.

A.1 $(M a P \& S a M) \supset S a P$

A.2 $(M e P \& S a M) \supset S e P$

A.3 $S e P \supset P e S$

A.4 $S a S$

A.5 SiP $\supset$ SiS

A.6 $S o P \supset S i S$

A.7 $S i P \equiv \neg S e P$ 


\section{A.8 $S o P \equiv \neg S a P$}

Единственное правило вывода - modus ponens.

Доказательство полноты можно найти в работе [1, с. 66-76].

Знаку общего имени может быть поставлено в соответствие понятие. Это нашло отражение в логической литературе, когда, говоря о субъекте и предикате атрибутивного высказывания, употребляют термин понятие, а не общее имя. С каждым понятием помимо объема мы связываем содержание как некоторую совокупность признаков, посредством которых выделяем мыслимые в понятии предметы. Благодаря этому появляется возможность анализировать связь между субъектом и предикатом атрибутивного высказывания не только с точки зрения объема понятия, но и с точки зрения его содержания, т.е. интенсионально. На это обращал внимание еще Лейбниц.

"Действительно, говоря: „Всякий человек есть животное“, я хочу этим сказать, что все люди находятся в числе всех животных, но одновременно я имею в виду, что идея животного включена в идею человека. Животное содержит больше индивидов, чем человек, но человек содержит больше идей или формальных определений. Животное содержит больше экземпляров, человек - больше степеней реальности; у первого больше оббем, у второго больше содержание. Поэтому мы вправе сказать, что все учение о силлогизме можно доказать на основании учения de continente et contento (о содержащем и содержимом), которое отлично от учения о челом и части» [6, т. 2, с. 501-502].

Аналогичные мысли можно встретить и у И. Канта, когда он рассуждает о различии между аналитическими и синтетическими суждениями $[4$, c. $37-38,132]$.

Строгое уточнение интенсиональной интерпретации атрибутивных высказываний было дано В. Маркиным в работах $[1,7,8,9]$.

\section{3 Интенсиональные модели}

ОПРЕДЕЛЕНИЕ 4. Итенсионалъной моделью для простых категорических атрибутивных высказываний будем называть четверку $\mathbf{I}=$ $\langle L, \Pi, H, \pi\rangle$, где

1. $L-$ непустое множество литералов $\left\{p_{1}, \sim p_{1}, p_{2}, \sim p_{2}, \ldots\right\}$, представляющих положительные и отрицательные признаки. Литерал без знака « » является положительным признаком, а литерал с этим знаком - отрицательным при знаком; 
2. $П-$ множество всех непустых подмножеств $L$ (множество содержаний всех понятий);

3. $H$ - множество всех непустых непротиворечивых подмножеств $L$, т.е. не содержащих одновременно $p_{i}$ и $\sim p_{i}$ для некоторого $i$ (множество всех непротиворечивых содержаний понятий).

4. $\pi$ - функция, определенная на множестве символов общих имен и сопоставляющая каждому из них некоторое содержание понятие $\pi(P) \in \Pi$.

В фундаментальной силлогистике $(\Phi С)$ значимость атрибутивных высказываний в интенсиональной модели I определяется следующим образом:

I.1 $\mathbf{I}=S a P \Longleftrightarrow \pi(P) \subseteq \pi(S)$ или $\pi(S) \notin H$

I.2 I $=S e P \Longleftrightarrow \pi(S) \cup \pi(P) \notin H$

I.3 I $=S i P \Longleftrightarrow \pi(S) \cup \pi(P) \in H$

I.4 $\mathbf{I}=S o P \Longleftrightarrow \pi(P) \backslash \pi(S) \neq \emptyset$ и $\pi(S) \in H$

Формула $A$ интенсионально общезначима, если и только если она значима в каждой интенсиональной модели I. Доказательство полноты можно найти в работе [1, с. 310-321].

Обратим внимание на то, что В. Маркин говорит не об истинности формул в модели, а об их значимости. Если придерживаться корреспондентской теории истинности, то из значимости высказывания SiP в интенсиональной модели вовсе не обязательно следует его истинность, поскольку значимость в данном случае означает всего лишь совместную непротиворечивость содержаний понятий $S$ и $P$, что является всего лишь необходимым условием истинности в корреспондентском смысле. В то же время интенсиональная значимость высказывания $\mathrm{SaP}$ является достаточным условием его истинности в корреспондентском смысле.

Поскольку значимость высказываний в интенсиональных моделях устанавливается без обращения к объемам понятий, они все являются аналитическими.

Предложенные В. Маркиным интенсиональные модели очень интересны и важны с историко-философской точки зрения, поскольку являются строгим уточнением идей, высказанных в разные эпохи разными 
философами. K сожалению, эти модели не получили должного отклика и развития в работах других логиков. Хотелось бы подчеркнуть, что именно результаты Маркина послужили толчком для построения предлагаемых ниже синтаксических моделей категорических атрибутивных высказываний.

Содержания понятий, с помощью которых устанавливается связь субъекта и предиката высказываний в интенсиональных моделях, не даны нам от Бога, а являются искусственными конструктами, посредством которых мы выделяем и связываем с общим именем мыслимые совокупности объектов. При этом мыслимые объекты вовсе не должны обладать реальным существованием. Простейшими примерами таких понятий являются понятия Кентавра, Пегаса или Олимпийского бога. Мы мыслим их исключительно интенсионально посредством набора признаков, составляющих содержание понятия. Но откуда берутся в содержании понятия эти признаки?

Ответ лежит на поверхности. Этому служат операции определения. Если вынести за скобки мотивацию принятия того или иного определения, сама эта операция является исключительно синтаксической. Приняв на уровне языка определение «Человек - это двуногое и бесперое животное», мы на уровне содержания связали с общим именем Человек два положительных признака Животное, Двуногое и один отрицательный - Не покрытый перъями. После этого общеутвердительное высказывание «Все люди - животные» становится аналитически истинным. Доказательство его аналитической истинности может быть проведено исключительно синтаксически, опираясь лишь на определения общих имен без каких-либо ссылок на содержания понятий. Это наблюдение и было положено в основу построения синтаксических моделей категорических атрибутивных высказываний.

Общеутвердительное высказывание $S a P$ синтаксически значимо, если и только если из дефиниенса определения субъекта $S$ можно дедуцировать дефиниенс определения предиката $P$.

Общеотрицательное высказывание $S e P$ синтаксически значимо, если и только если дефиниенсы определений субъекта $S$ и предиката $P$ несовместимы друг с другом.

Частноутвердительное высказывание $S i P$ синтаксически значимо, если и только если дефиниенсы определений субъекта $S$ и предиката $P$ совместимы друг с другом. 
Частноотрицательное высказывание $S o P$ синтаксически значимо, если и только если дефиниенс определения субъекта $S$ совместим с отрицанием дефиниенса определения предиката $P$.

\section{4 Синтаксические модели}

ОПРЕДЕЛЕНИЕ 5. Синтаксической моделью для простых атрибутивных высказываний будем называть пятерку $\mathbf{S}=\langle L, C, F, \vdash, \delta\rangle$, где

1. $L-$ непустое множество исходных символов для представления признаков $\left\{p_{1}, p_{2}, \ldots\right\}$;

2. $C-$ множество связок. Пусть это будет привычный набор $\{\wedge, \vee, \sim\}$

3. $F-$ множество всех формул логики высказываний в языке с исходными символами $L \cup C$;

4. $\vdash$ - отношение выводимости на $F$, в качестве которого возьмем отношение выводимости классической логики высказываний;

5. $\delta$ - функция, определенная на множестве символов общих имен и сопоставляющая каждому из них некоторую формулу $\delta(P) \in F$ (дефиниенс явного определения общего имени $P$ ).

Напомним некоторые из свойств отношения выводимости классической логики высказываний.

C.1 $A \vdash A$

C.2 $\Gamma, A, B, \Delta \vdash C \Longrightarrow \Gamma, B, A, \Delta \vdash C$

C.3 $\Gamma, A, A, \Delta \vdash C \Longrightarrow \Gamma, A, \Delta \vdash C$

C.4 $\Gamma, A, \Delta \vdash C \Longrightarrow \Gamma, A, B, \Delta \vdash C$

C.5 $\Gamma \vdash A ; A, \Delta \vdash B \Longrightarrow \Gamma, \Delta \vdash B$

C.6 $\Gamma, A, B, \Delta \vdash C \Longrightarrow \Gamma, A \wedge B, \Delta \vdash C$

C.7 $\Gamma, A \wedge B, \Delta \vdash C \Longrightarrow \Gamma, A, B, \Delta \vdash C$

C.8 $\Gamma \vdash A ; \Gamma \vdash B \Longrightarrow \Gamma \vdash A \wedge B$

C.9 $\Gamma \vdash A \wedge B \Longrightarrow \Gamma \vdash A$ 


\section{C.10 $\Gamma \vdash A \wedge B \Longrightarrow \Gamma \vdash B$}

C.11 $\Gamma, A, \sim A \vdash B$

В последующем изложении факт существования для некоторой формулы $A$ выводимости $\Gamma \vdash A \wedge \sim A$ будем обозначать посредством $\Gamma \vdash \mathbf{f}$.

В фундаментальной силлогистике $(\Phi \mathrm{C})$ значимость атрибутивнъх высказываний в синтаксической модели $\mathbf{S}$ определяется следующим образом:

$\mathbf{S} .1 \mathbf{S} \models S a P \Longleftrightarrow \delta(S) \vdash \delta(P)$

$\mathbf{S . 2} \mathbf{S} \models S e P \Longleftrightarrow \delta(S), \delta(P) \vdash \mathbf{f}$

$\mathbf{S . 3} \mathbf{S}=S i P \Longleftrightarrow \delta(S), \delta(P) \nvdash \mathbf{f}$

S.4 $\mathbf{S}=S o P \Longleftrightarrow \delta(S) \nvdash \delta(P)$

Формула $A$ синтаксически общезначима, если и только если она значима в каждой синтаксической модели $\mathbf{S}$.

Покажем, что синтаксические модели являются адекватными для фундаментальной силлогистики.

Теорема 1. Теорема непротиворечивости. Всякая теорема фундаментальной силлогистики значима в каждой синтаксической модели.

\section{ДоказАТЕЛЬСТво.}

Пусть нам дана синтаксическая модель $\mathbf{S}=\langle L, C, F, \vdash, \delta\rangle$. Проверим, что каждая из аксиом фундаментальной силлогистики значима в ней.

A.1 $(M a P \& S a M) \supset S a P$

1. $\delta(M) \vdash \delta(P) \quad$ - допущение

2. $\delta(S) \vdash \delta(M) \quad$ - допущение

3. $\delta(S) \vdash \delta(P) \quad$ - из 1,2 по С.5

A.2 $(M e P \& S a M) \supset S e P$

1. $\delta(M), \delta(P) \vdash \mathbf{f} \quad$ - допущение

2. $\delta(S) \vdash \delta(M) \quad$ - допущение

3. $\delta(S), \delta(P) \vdash \mathbf{f} \quad$ - из 1,2 по C.5

A.3 $S e P \supset P e S$ 
1. $\delta(S), \delta(P) \vdash \mathbf{f} \quad$ - допущение

2. $\delta(P), \delta(S) \vdash \mathbf{f} \quad$ - из 1 по С.2

\section{A.4 $S a S$}

1. $\delta(S) \vdash \delta(S) \quad$ - по С. 1

A.5 $S i P \supset S i S$

1. $\delta(S), \delta(P) \nvdash \mathbf{f} \quad$ - допущение

2. $\delta(S), \delta(S) \vdash \mathbf{f} \quad$ - допущение от противного

3. $\delta(S) \vdash \mathbf{f} \quad$ - из 2 по С. 3

4. $\delta(S), \delta(P) \vdash \mathbf{f} \quad-$ из 3 по С.4

5. противоречие $-1,4$

A.6 $S o P \supset S i S$

1. $\delta(S) \nvdash \delta(P) \quad$ - допущение

2. $\delta(S), \delta(S) \vdash \mathbf{f} \quad$ - допущение от противного

3. $\delta(S) \vdash \mathbf{f} \quad$ - из 2 по С.3

4. $\delta(S) \vdash A \wedge \sim A \quad$ - из 3 по определению $\mathbf{f}$

5. $A, \sim A \vdash \delta(P) \quad-$ C.11

6. $A \wedge \sim A \vdash \delta(P) \quad$ - из 5 по С. 6

7. $\delta(S) \vdash \delta(P) \quad$ - из 4,6 по С.5

8. противоречие $-1,7$

A.7 $S i P \equiv \neg S e P$ - пункты 2,3 определения значимости в синтаксической модели.

A.8 $S o P \equiv \neg S a P$ - пункты 1,4 определения значимости в синтаксической модели.

Все аксиомы логики высказываний значимы в синтаксической модели, а modus ponens сохраняет это свойство.

Теорему о полноте фундаментальной силлогистики относительно синтаксических моделей докажем методом Хенкина.

Обычным образом любое непротиворечивое множество формул мы можем расширить до максимального ФС-непротиворечивого $\Delta$, обладающего свойствами:

M.1 $\Delta$ содержит все теоремы ФС. 
M.2 $\Delta$ замкнуто относительно modus ponens.

M.3 $\neg A \in \Delta \Longleftrightarrow A \notin \Delta$.

M.4 $A \& B \in \Delta \Longleftrightarrow A \in \Delta$ и $B \in \Delta$.

M.5 $A \vee B \in \Delta \Longleftrightarrow A \in \Delta$ или $B \in \Delta$.

M.6 $A \supset B \in \Delta \Longleftrightarrow A \notin \Delta$ или $B \in \Delta$.

Поскольку для доказательства теоремы полноты нам понадобится рассматривать максимально непротиворечивые множества, полученные в результате расширения конечных множеств формул, примем, что множество исходных символов общих имен конечно.

Для максимально ФС-непротиворечивого множества $\Delta$ определим каноническую синтаксическую модель $\mathbf{S}_{\Delta}=\left\langle L, C, F, \vdash, \delta_{\Delta}\right\rangle$ следующим образом.

ОПРЕДЕЛЕНИЕ 6.

1. $L$ будет состоять из символов общих имен языка $\{S, P, M, Q, R, N, \ldots\}$.

2. $C=\{\wedge, \vee, \sim\}$.

3. $F-$ множество всех формул логики высказываний в языке с исходными символами $L \cup C$.

4. $\vdash$ - классическое отношение выводимости на $F$.

5. $\delta_{\Delta}$ определим следующим образом. Пусть $\Omega_{Q}=\{T: Q a T \in \Delta\} \cup$ $\{\sim T: Q e T \in \Delta\}$. Обозначим посредством $\Omega_{Q \wedge}$ элементарную конъюнкцию всех элементов $\Omega_{Q}$. Поскольку мы ограничились языком с конечным набором символов общих имен, эта конъюнкция конечна. Тогда $\delta_{\Delta}(Q)=\Omega_{Q \wedge}$. Это определение в значительной степени опирается на идеи В. Маркина для канонических интенсиональных моделей.

Поскольку для любого общего имени $S$ нашего языка формула $S a S$ является теоремой $\Phi$ и и принадлежит каждому максимальному $\Phi С-$ непротиворечивому множеству $\Delta$, т.е. $S \in \Omega_{S}$, то $\delta_{\Delta}(S)$ всегда определена.

Единственным нетривиальным шагом в доказательстве теоремы о полноте является следующая лемма. 
Лемма 1. Для произвольного максимального ФС-непротиворечивого множества $\Delta$ и произвольной формулы $A$ верно, что $A \in \Delta \Longleftrightarrow$ $S_{\Delta} \models A$.

\section{ДоКАЗАТЕЛЬСТво.}

Рассмотрим четыре базисных случая.

I. $A$ есть $S a P$

Докажем $S a P \in \Delta \Longrightarrow \mathbf{S}_{\Delta}=S a P$

$\begin{array}{ll}\text { 1. } S a P \in \Delta & \text { - допущение } \\ \text { 2. } T \in \Omega_{P} & \text { - допущение } \\ \text { 3. } P a T \in \Delta & \text { - из } 2 \text { по определению } \delta_{\Delta} \\ \text { 4. }(P a T \& S a P) \supset S a T & \text { - А.1 } \\ \text { 5. } S a T \in \Delta & \text { - из } 1,3,4 \text { по М.2, М.4 } \\ \text { 6. } T \in \Omega_{S} & \text { - из } 5 \text { по определению } \delta_{\Delta} \\ \text { 7. } \sim T \in \Omega_{P} & \text { - допущение } \\ \text { 8. } P e T \in \Delta & \text { - из } 7 \text { по определению } \delta_{\Delta} \\ \text { 9. }(P e T \& S a P) \supset S e T & \text { - А.2 } \\ \text { 10. } S e T \in \Delta & \text { - из } 1,8,9 \text { по М.2, М.4 } \\ \text { 11. } \sim T \in \Omega_{S} & \text { - из } 10 \text { по определению } \delta_{\Delta} \\ \text { 12. } \delta_{\Delta}(S) \vdash \delta_{\Delta}(P) & \text { - из } 1,2-6,7-11 \text { по С.8, С.9 } \\ \text { 13. } S_{\Delta}=S a P & \text { - из } 12 \text { по S.1 }\end{array}$

Докажем $\mathbf{S}_{\Delta}=S a P \Longrightarrow S a P \in \Delta$

1. $\mathbf{S}_{\Delta}=S a P$

2. $\delta_{\Delta}(S) \vdash \delta_{\Delta}(P)$

3. $\delta_{\Delta}(S) \nvdash \mathbf{f}$

4. $\Omega_{S \wedge} \vdash \Omega_{P \wedge}$

5. $\Omega_{P} \subseteq \Omega_{S}$

6. $P a P \in \Delta$

7. $P \in \Omega_{P}$

8. $P \in \Omega_{S}$

9. $S a P \in \Delta$

10. $\delta_{\Delta}(S) \vdash \mathbf{f}$

11. $\Omega_{S \wedge} \vdash \mathbf{f}$

12. $T \in \Omega_{S}, \sim T \in \Omega_{S}$

13. $S a T \in \Delta, S e T \in \Delta$
- допущение

- из 1 по S.1

- допущение

- из 2

- из 3,4 по С. 8, С.9

- A.4, M.1

- из 6 по определению $\Omega_{P}$

- из 5,7

- из 8 по определению $\Omega_{S}$

- допущение

- из 10 по определению $\delta_{\Delta}(S)$

- из 11 для некоторого Т

- из 12 по определению $\Omega_{S}$ 


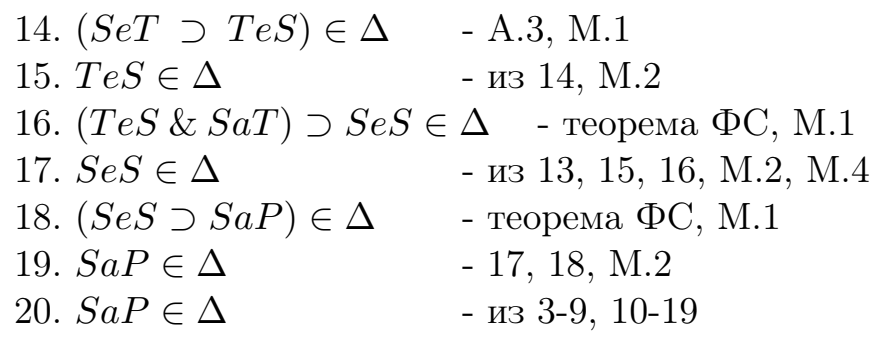

\section{II. $A$ есть $S e P$}

Докажем $\mathrm{SeP} \in \Delta \Longrightarrow \mathbf{S}_{\Delta} \models S e P$

1. $S e P \in \Delta \quad$ - допущение

2. $(S e P \supset P e S) \in \Delta \quad$ - A.3, M.1

3. $P e S \in \Delta \quad$ - из 1,2, M.2

4. $S a S \in \Delta \quad$ - A.4, M.1

5. $\sim S \in \Omega_{P} \quad$ - из 3 по определению $\Omega_{P}$

6. $S \in \Omega_{S} \quad$ - из 4 по определению $\Omega_{S}$

7. $\Omega_{S \wedge}, \Omega_{P \wedge} \vdash S \wedge \sim S \quad$ - из 5,6

8. $\delta_{\Delta}(S), \delta_{\Delta}(P) \vdash \mathbf{f} \quad-$ из 7 по определению $\delta_{\Delta}, \mathbf{f}$

9. $\mathbf{S}_{\Delta}=S e P \quad$ - из 8 по $\mathrm{S} .2$

$$
\text { Докажем } \mathbf{S}_{\Delta}=S e P \Longrightarrow S e P \in \Delta
$$

1. $\mathbf{S}_{\Delta}=S e P \quad$ - допущение

2. $\delta_{\Delta}(S), \delta_{\Delta}(P) \vdash \mathbf{f} \quad$ - из 1 по $\mathrm{S} .2$

3. $\delta_{\Delta}(S) \vdash T \wedge \sim T$ или

$\delta_{\Delta}(P) \vdash T \wedge \sim T$ или

$\left[\delta_{\Delta}(S) \vdash T, \delta_{\Delta}(P) \vdash \sim T\right]$ или

$\left[\delta_{\Delta}(S) \vdash \sim T, \delta_{\Delta}(P) \vdash T\right] \quad$ - из 2

4. $\delta_{\Delta}(S) \vdash T \wedge \sim T$ - допущение

5. $\Omega_{S \wedge} \vdash T \wedge \sim T \quad$ - из 4 по определению $\delta_{\Omega}$

6. $T \in \Omega_{S}, \sim T \in \Omega_{S} \quad$ - из 5 по определению $\Omega_{S \wedge}$

7. $S a T \in \Delta \quad$ - из 6 по определению $\Omega_{S}$

8. $S e T \in \Delta \quad$ - из 6 по определению $\Omega_{S}$

9. $(S e T \supset T e S) \in \Delta \quad-$ A.3, M.1

10. $T e S \in \Delta \quad$ - из 8, 9, М.2

11. $(T e S \& S a T) \supset S e S \in \Delta \quad$ - A.2, M.1

12. $S e S \in \Delta \quad$ - из $7,10,11$, M.2, М.4 


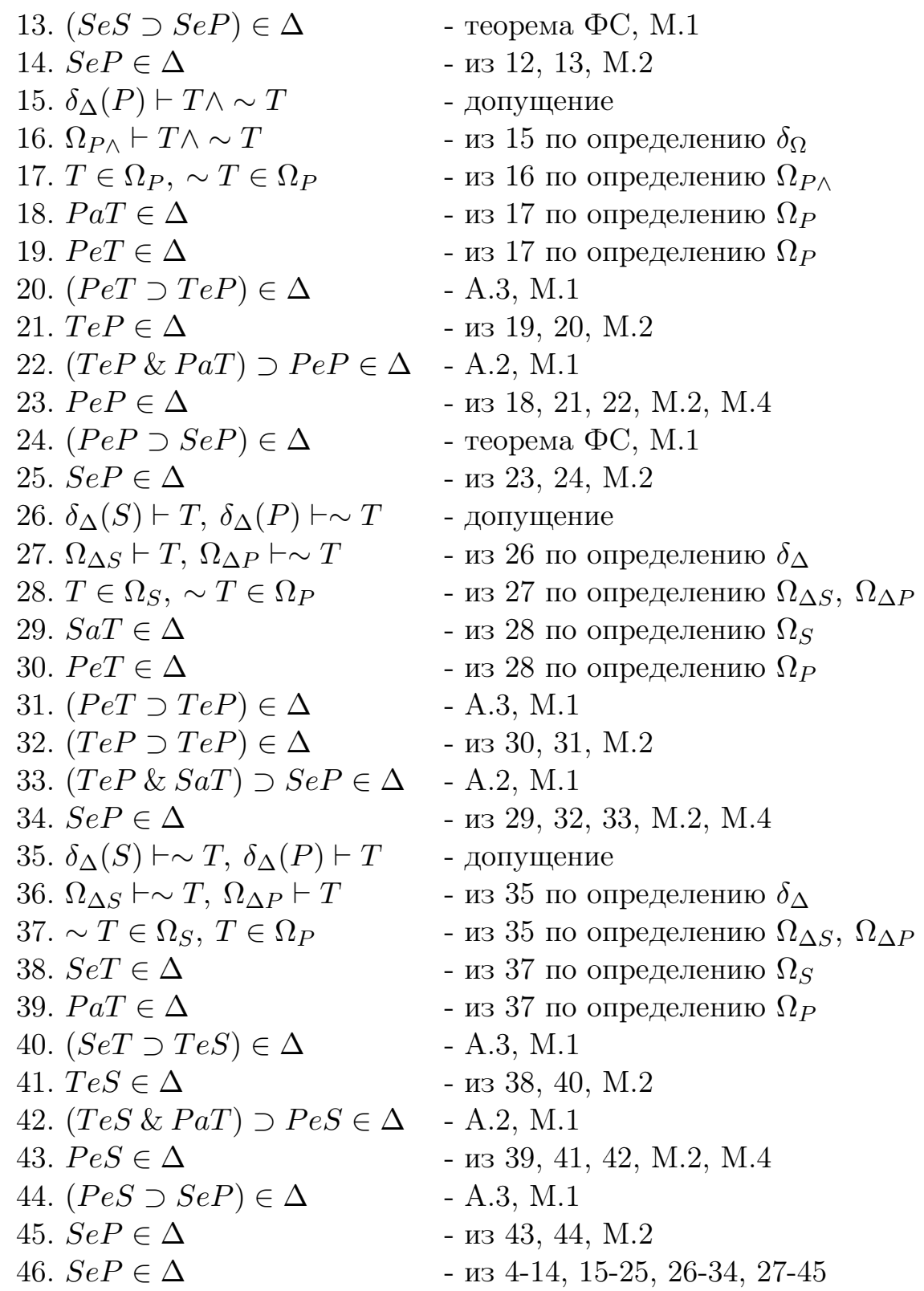

III. $A$ есть $S i P$

В силу аксиомы $S i P \equiv \neg S e P$, условий значимости в синтаксической модели S.2 и S.3, а также свойства М.3 максимальных ФС- 
непротиворечивых множеств данный случай сводится к случаю для SeP.

IV. $A$ есть $S o P$

В силу аксиомы $S o P \equiv \neg S a P$, условий значимости в синтаксической модели S.1 и S.4, а также свойства М.3 максимальных ФCнепротиворечивых множеств данный случай сводится к случаю для SaP.

Оставшиеся случаи для логических связок рассматриваются стандартно на основе свойств M.1-M.6 максимальных ФСнепротиворечивых множеств.

Теорема 2. Теорема о полноте. Если формула А синтаксически общезначима, то она доказуема в фундаментальной силлогистике.

\section{ДокАЗАТЕЛЬСТво.}

Допустим, формула $A$ значима во всех синтаксических моделях, но не доказуема. Тогда формула $\neg A$ непротиворечива. Множество $\{\neg A\}$ может быть расширено до максимального ФС-непротиворечивого $\Delta$. В канонической синтаксической модели $\mathbf{S}_{\Delta}$ будет иметь место $\mathbf{S}_{\Delta} \models \neg A$ и, следовательно, $\mathbf{S}_{\Delta} \not \models A$, что противоречит допущению о значимости формулы $A$ во всех синтаксических моделях.

\section{5 Заключительные замечания}

I. В настоящей работе мы рассмотрели три вида интерпретаций простых позитивных категорических атрибутивных высказываний. Относительно каждой из них имеет место теорема о полноте фундаментальной чистой позитивной силлогистики [1, с. 31-32, 66-76]. Данный вид высказываний и фундаментальная силлогистика выбраны по той причине, что они лежат в начале целого ряда языков атрибутивных высказываний и соответствующих им логических систем силлогистического типа.

Экстенсиональные интерпретации других систем чистой позитивной силлогистики отличаются друг от друга условиями на пустоту или непустоту терминов в тех или иных высказываниях. Эти системы представляют историко-логический интерес с точки зрения изучения взглядов разных философов, но с логической точки зрения они плохо обоснованы. На их фоне выделяется система традиционной силлогистики $[1$, 
с. 32-34, 90-95], которая по классу теорем совпадает с чистой позитивной силлогистикой Лукасевича и системой С4 В.А. Смирнова. Адекватные синтаксические модели для этой системы силлогистики получаются вполне естественно. Одним из методологических требований к определению предикатных констант является непротиворечивость [5, с. 15-18]. Если в соответствии с этим требованием наложить на функцию $\delta$ ограничение, чтобы она сопоставляла общим именам лишь непротиворечивые формулы, то полученные синтаксические модели будут моделями традиционной силлогистики.

II. При построении синтаксических моделей мы допустили, что в определениях общих имен в качестве дефиниенсов им могут быть сопоставлены любые формулы логики высказываний, но при доказательстве теоремы о полноте оказалось достаточным ограничиться лишь элементарными конъюнкциями. В то же время очевидно, что синтаксические модели могут быть построены не только для простых категорических атрибутивных высказываний, но и для высказываний с отрицательными и сложными терминами. В синтаксической модели изменения затронут лишь определение функции $\delta$, которая сложным терминам будет сопоставлять те или иные комбинации дефиниенсов, зависящие от вида сложного термина, но не коснутся определения значимости в модели.

Если мы попробуем построить для атрибутивных высказываний со сложными терминами интенсиональную модель, то это можно сделать, но потребуется ввести как минимум некоторую алгебру смыслов, что ухудшит интуитивную прозрачность интерпретации.

III. В зависимости от решаемых задач параметры синтаксической модели $\mathbf{S}=\langle L, C, F, \vdash, \delta\rangle$ могут варьироваться. Например, мы можем варьировать $L$, взяв вместо простого множества признаков множество предикатных символов, чтобы формулировать определения понятий не в языке логики высказываний, а в языке логики предикатов. При необходимости мы можем изменить множество связок $C$, изменив тем самым и множество формул $F$.

Отношение выводимости $\vdash$ также допускает варьирование. Вместо выводимости в классической логике мы можем взять выводимость в интуиционистской, релевантной, паранепротиворечивой или модальной логике. Коль скоро упомянута модальная логика, ничто не мешает построить синтаксические модели для модальной силлогистики, напрямую связав известные виды модальностей с модальностями атрибутивных высказываний. 
При определении синтаксической модели мы приняли соглашение обозначать выводимость $\Gamma \vdash A \wedge \sim A$ посредством $\Gamma \vdash \mathbf{f}$, где $\mathbf{f}$ представляет противоречие (ложь, нежелательную ситуацию). В различных логических системах обнаружение противоречия или аналогичной ситуации может пониматься по-разному. Например, в системе релевантной логики $R$ есть две различных константы для представления лжи $\mathbf{f}$ и $\mathbf{F}$, а в паранепротиворечивых логиках получение противоречия не является критическим.

IV. Определенный интерес для философов и логиков должно представлять то, что синтаксическая интерпретация атрибутивных высказываний свободна от онтологических допущений. Если мы хотим осмысленно рассуждать о греческих богах, нет никакой необходимости предполагать, что они существуют в каком-то выдуманном мире и строить столь же выдуманную экстенсиональную модель. Достаточно принять подходящие определения используемых понятий и рассуждать о них, нисколько не заботясь о фактической пустоте объемов этих понятий. Все значимые утверждения о греческих богах и кентаврах мы получим исключительно на основе определений, которые им дали.

Стремление для каждой логической системы или теории иметь экстенсиональную модель оправдано лишь традицией, когда наши утверждения относились лишь к макромиру - «Сократ - человек», «Все лебеди - белые». В процессе познания в научный обиход вводятся абстрактные понятия и понятия, которые не имеют явных коррелятов в реальном мире. Требование построения для них экстенсиональных моделей является ограничением, которое зачастую лишь затрудняет работу. Достаточно вспомнить историю введения в научный обиход комплексных чисел. С ними смирились лишь тогда, когда им была дана наглядная геометрическая интерпретация, хотя практическая польза от включения их в язык математики проявилась гораздо раньше.

Синтаксические модели атрибутивных высказываний позволяют поновому подойти к построению некоторых логик, например, логики оценок. В логике абсолютных оценок Добро и Зло рассматриваются как модальные операторы [3]. Смысл их определяется неявно посредством подходящих аксиом модальной логики. Построить адекватную и содержательно приемлемую экстенсиональную модель для таких логик довольно трудно. В то же время синтаксические модели для логик абсолютных оценок вполне возможны.

V. Если вспомнить семантический треугольник и соотнести его элементы с экстенсиональной, интенсиональной и синтаксической моделя- 
ми силлогистики, то окажется, что силлогистика обладает удивительным свойством - она полна на уровне значений общих имен, полна на уровне их смыслов и полна на уровне синтаксиса. Это необычное свойство силлогистики, которое может быть названо семиотической полнотой, заслуживает осмысления.

\section{Литература}

[1] Бочаров В.А., Маркин В.И. Силлогистические теории. М.: ПрогрессТрадиция, 2010. $336 \mathrm{c.}$

[2] Гилъберт Д., Аккерман В. Основы теоретической логики. М.: ИЛ, 1947. $304 \mathrm{c}$.

[3] Ивин А.А. Логика оценок и норм. М.: Проспект, 2015.

[4] Кант И. Критика чистого разума. М.: Мысль, 1994. 591 с.

[5] Карпович B.H. Термины в структуре теорий (логический анализ). Новосибирск: Наука, 1978. 128 с.

[6] Лейбнии Г.В. Сочинения в четырех томах. М.: Мысль, 1984.

[7] Маркин В.И Интенсиональная семантика традиционной силлогистики // Логические исследования. Вып. 8. М.: Наука, 2001. С. 82-91.

[8] Маркин В.И Фундаментальная силлогистика с интенсиональной точки зрения // Логические исследования. Вып. 9. М.: Наука, 2002. С. 119-130.

[9] Маркин В.И Интенсиональная семантика для систем позитивной силлогистики // Логика и В.Е.К. М.: Современные тетради, 2003. С. 166-174. 


\title{
V.I. SHALACK \\ Syntactic Interpretation of Categorical Attributive Propositions
}

\author{
Shalack Vladimir Ivanovich \\ Department of Logic, Institute of Philosophy, Russian Academy of Sciences. \\ Volkhonka 14/5, Moscow, 119991, Russian Federation. \\ e-mail: shalack@gmail.com
}

In the article we propose a new type of models for categorical attributive propositions. Usually the subject and predicate of the attributive proposition are interpreted extensionally as some sets of individuals. Logical relations between subject and predicate are understood as the set-theoretic relations between their extensions. We propose a syntactic interpretation of subject and predicate of attributive propositions and interpret them as some formulas of propositional logic. These formulas can be understood as the definitions of common terms. Logical relations between subject and predicate are understood as the logical relations between their definitions. The article contains a proof that the system of fundamental syllogistic is consistent and complete with respect to the proposed semantics. Built interpretation can be generalized to other systems of syllogistic.

Keywords: logic, attributive proposition, common term, syllogistic, definition, concept, analyticity, model

\section{References}

[1] Bocharov, V.A., Markin, V.I. Sillogisticheskie teorii [Syllogistic theories]. M.: Progress-Tradicija, 2010. 336 p. (In Russian)

[2] Gil'bert, D., Akkerman, V. Osnovy teoreticheskoj logiki [Foundations of theoretical logic]. M.: IL, 1947. 304 p. (In Russian)

[3] Ivin, A.A. Logika ocenok $i$ norm [Logic assessments and standards]. M.: Prospekt, 2015. (In Russian)

[4] Kant, I. Kritika chistogo razuma [Critique of Pure Reason]. M.: Mysl', 1994. 591 p. (In Russian)

[5] Karpovich, V.N. Terminy v strukture teorij (logicheskij analiz) [The terms in the structure of theories (logical analysis)]. Novosibirsk: Nauka, 1978. 128 p. (In Russian)

[6] Lejbnic, G.V. Sochinenija v chetyreh tomah [Works in four volumes]. M.: Mysl', 1984. (In Russian)

[7] Markin, V.I. "Intensional'naja semantika tradicionnoj sillogistiki" [Intensional semantics of traditional syllogistic], Logicheskie issledovanija [Logical Investigations]. M.: Nauka, 2001, vol. 8, pp. 82-91. (In Russian) 
[8] Markin, V.I. "Fundamental'naja sillogistika s intensional'noj tochki zrenija" [Fundamental syllogistics with intensional point of view], Logicheskie issledovanija [Logical Investigations]. M.: Nauka, 2002, vol. 9, pp. 119-130. (In Russian)

[9] Markin, V.I. "Intensional'naja semantika dlja sistem pozitivnoj sillogistiki" [Intensional semantics for systems of positive syllogistic], Logika $i$ V.E.K. [Logic and V.E.K.]. M.: Sovremennye tetradi, 2003, pp. 166-174. (In Russian) 